\title{
MVF-HER-2(597-626)/MVF-HER-2 (266-296) Peptide Vaccine
}

National Cancer Institute

\section{Source}

National Cancer Institute. MVF-HER-2(597-626)/MVF-HER-2 (266-296) Peptide Vaccine.

NCI Thesaurus. Code C97344.

A combination peptide vaccine of 2 chimeric peptides of the promiscuous $T$ cell epitope derived from measles virus fusion protein (MVF; amino acid residues 288-302) cosynthesized with B-cell epitopes derived from the HER-2/neu a.a. 597-626 and HER2/neu a.a. 266-296, with potential antineoplastic activity. Vaccination with MVF-HER2(597-626)/MVF-HER-2(266-296) peptide vaccine may be capable of inducing an active specific immune response, mounting a cytotoxic T-lymphocyte $(C T L)$ response and an antibody-dependent cell-mediated cytotoxicity (ADCC) ag ainst tumor cells that overexpress the HER-2 protein. The oncogenic protein HER-2, a member of the human epidermal growth factor receptor (EGFR) family of tyrosine kinases, is overexpressed in a variety of cancers and is correlated with increased tumor growth, progression and a poor prognosis. HER-2(597-626) corresponds to the binding site of trastuzumab on the extracellular domain IV of HER-2; HER-2 (266-296) corresponds to the binding site of pertuzumab on the dimerization loop of domain II of HER-2. 